\title{
The Metal-Halide Lamp Under Varying Gravity Conditions Measured by Emission and Laser Absorption Spectroscopy
}

\author{
A. J. Flikweert - T. Nimalasuriya - G. M. W. Kroesen • \\ M. Haverlag • W. W. Stoffels
}

Received: 4 February 2008 / Accepted: 7 January 2009 / Published online: 30 January 2009

(C) The Author(s) 2009. This article is published with open access at Springerlink.com

\begin{abstract}
Diffusive and convective processes in the metal-halide lamp cause an unwanted axial colour segregation. Convection is induced by gravity. To understand the flow phenomena in the arc discharge lamp it has been investigated under normal laboratory conditions, micro-gravity (ISS and parabolic flights) and hyper-gravity (parabolic flights $2 g$, centrifuge $1 g-10 g$ ). The measurement techniques are webcam imaging, and emission and laser absorption spectroscopy. This paper aims to give an overview of the effect of different artificial gravity conditions on the lamp and compares the results from the three measurement techniques.
\end{abstract}

Keywords Metal-halide lamp •

Emission spectroscopy •

Laser absorption spectroscopy • Artificial gravity

Electronic supplementary material The online version of this article (doi:10.1007/s12217-009-9106-z) contains

supplementary material, which is available to authorized users.

A. J. Flikweert ( $\varangle)$ · T. Nimalasuriya - G. M. W. Kroesen

M. Haverlag · W. W. Stoffels

Department of Applied Physics,

Eindhoven University of Technology, Na 2.15, PO Box 513,

5600 MB Eindhoven, The Netherlands

e-mail: a.flikweert@fz-juelich.de

W. W. Stoffels

e-mail:w.w.stoffels@tue.nl

Present Address:

A. J. Flikweert

IEF-5, Forschungszentrum Jülich, 52425 Jülich, Germany

\section{Introduction}

Worldwide, $20 \%$ of all electricity is used for lighting (International Energy Agency 2006; Stoffels et al. 2007). For this reason, efficient lamps are economically and ecologically important. Of all lamps, the well-known incandescent lamp has one of the worst efficiencies of about $4 \%$. Compact fluorescent lamps (also known as energy-saving lamps) and fluorescent tubes have better efficiencies of about $20 \%-30 \%$. One of the properties is the colour rendering index of a lamp. The well-known low-pressure sodium lamp (the deep orange street lighting) is very efficient, but the colour rendering is poor because of the monochromatic light.

High intensity discharge (HID) lamps are efficient lamps with a good colour rendering index. They are high pressure lamps based on arc emission (Stormberg 1981; Rogoff et al. 1987; Zollweg 1975; Dakin and Shyy 1989; Dakin et al. 1989; Lister et al. 2004; Bonvallet and Lawler 2003). These lamps are mainly used for outdoor applications; examples are shop lighting, street lighting, flood lighting of sport stadiums and city beautification. HID lamps have a high efficiency (up to $40 \%$ ) (Lister et al. 2004) and emit white light.

The most common HID lamp these days is the metalhalide (MH) lamp. It contains a buffer gas (usually mercury) and additives that act as the prime radiator in the visible. These additives increase the efficiency and the colour rendering. The additive is dosed as a salt; in our case we use dysprosium iodide $\left(\mathrm{DyI}_{3}\right)$.

When the lamp is burning vertically, segregation of the additives occurs (Fischer 1976; Stoffels et al. 2006) and colour segregation appears. This limits the lamp design for application in for example shop lighting. 


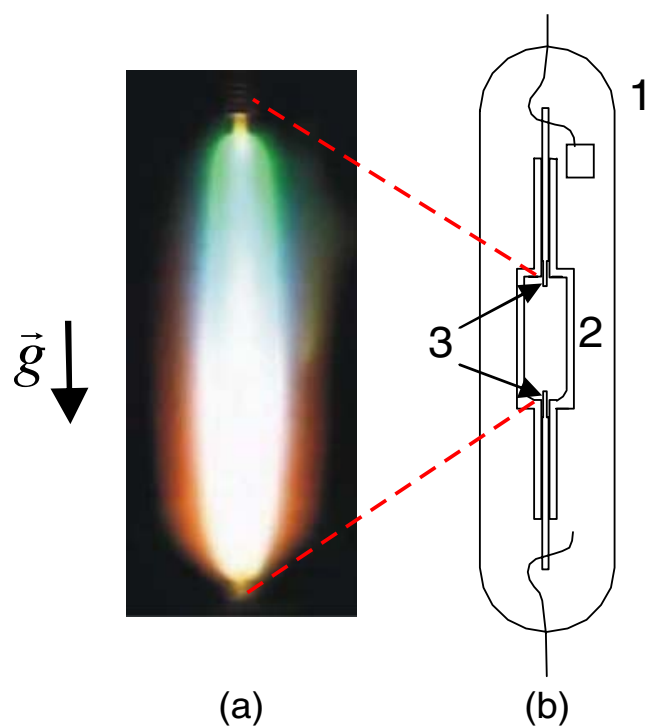

Fig. 1 a The burner of a MH lamp (contains $10 \mathrm{mg} \mathrm{Hg}$ and $4 \mathrm{mg}$ $\mathrm{DyI}_{3}$ ), colour segregation is clearly visible. b Schematic picture of the lamp, (1) outer bulb; (2) burner (size $8 \mathrm{~mm} \times 20 \mathrm{~mm}$ ); (3) electrodes, distance between both electrodes $\sim 16 \mathrm{~mm}$

Figure 1 shows a schematic drawing of the lamp and an image of the plasma when the lamp is burning. The bluish-white light at the bottom is caused by Dy atoms, whereas the bluish colour at the top is caused by $\mathrm{Hg}$ atoms. This non-uniform light output has a bad influence on the efficiency and the colour rendering of the lamp. The distribution of the Dy atoms is determined by convection and diffusion in the lamp. Convection is caused by gravity and to gain more insight in the flow phenomena, the lamp has been investigated under different gravity conditions. Previous efforts to observe the arc and its characteristics in micro-gravity include emission spectroscopy in the space shuttle (Rogoff et al. 1985; Bellows et al. 1984, 1985).

Besides lab experiments (1 $g$ ) (Flikweert et al. 2005; Nimalasuriya et al. 2005, 2006; Nimalasuriya 2007) we investigated the lamp during parabolic flights. Here the lamp was measured during periods of about 20 s of micro-gravity $(0 g)$ and hyper-gravity $(\sim 1.8 g)$ (Flikweert et al. 2006). In addition, the lamp was investigated in the International Space Station ISS (microgravity $0 g$ ) (Nimalasuriya 2007; Nimalasuriya et al. 2006). Finally the lamp was placed in a centrifuge $(1 g-10 g)$ (Nimalasuriya 2007; Nimalasuriya et al. 2007; Flikweert et al. 2007, 2008). This centrifuge is used as a tool to vary the (artificial) gravity and thus the amount of convection for a longer time than at the parabolic flights to assure stable arc conditions. The experimental methods to investigate the lamp are emission spectroscopy, Imaging Laser Absorption Spectroscopy (ILAS) (Flikweert et al. 2007) and webcam imaging.
This paper will give an overview of the artificial gravity used to vary the convection in the lamp and the measurement techniques to investigate the lamp. First, the theory of the transport processes in the $\mathrm{MH}$ lamp will be summarized. Next, the parabolic flight measurements and centrifuge measurements will be discussed, with the main focus on the centrifuge. After a system overview of the centrifuge is given, the three measurement techniques and some results will be discussed: webcam imaging, emission spectroscopy and ILAS measurements. Finally we want to draw some conclusions and give an outlook for future work.

\section{Transport Processes in the MH Lamp}

The theory of the transport processes in the MH lamp has been described in prior publications (Fischer 1976; Flikweert et al. 2005). The most important part will be summarized here. The lamp we use is a cost reference lamp (Fig. 1a) (Stoffels et al. 2006) and contains $300 \mathrm{mbar} \mathrm{Ar} / \mathrm{Kr}^{85}$ as starting gas, 5 or $10 \mathrm{mg} \mathrm{Hg}$ as buffer gas (which corresponds to a $\mathrm{Hg}$ pressure of about 6 and 12 bar) and $4 \mathrm{mg} \mathrm{DyI}{ }_{3}$ as salt additive (the vapour is saturated; pressure $\sim 100 \mathrm{~Pa}$ at the cold spot). When the lamp is ignited, the $\mathrm{Hg}$ evaporates and a mercury discharge is created. The lamp heats up and the salt additive enters the arc discharge. The centre of the arc is much hotter $(\sim 5500 \mathrm{~K})$ than the wall $(\sim 1200 \mathrm{~K})$ (Zhu 2005). First we have convection because of this large gradient. Convection is induced by gravity. The hot gas in the centre moves upward, whereas the colder gas near the wall moves downward. Next, the temperature gradient causes a chemical equilibrium. At the wall mainly molecules are present, whereas in the centre mainly Dy atoms are present. In addition, because of the high temperature, Dy atoms are ionized in the very centre. Due to the different diffusion velocities of the heavier molecules and the lighter atoms and ions, the molecules move slower inward than the atoms and ions move outward. This causes a hollow radial pressure profile of atoms; this is called radial segregation.

While the Dy atoms are moving outward and get closer to the wall, they are dragged downward by the convection. In this way the Dy atoms stay mainly at the bottom of the lamp. The combination of convection and radial segregation thus causes axial segregation in the lamp as seen in Fig. 1a. The representative flow lines of the atomic dysprosium are drawn in Fig. 2.

Axial segregation of Dy is thus caused by the combination of the convection and diffusion. When these effects are in the same order of magnitude, maximal axial segregation occurs. In the limiting cases, when the 
Fig. 2 Schematic of the flow lines of atomic dysprosium in the burner. Thick lines represent a high dysprosium density whereas thin lines represent a low density (Flikweert et al. 2005)
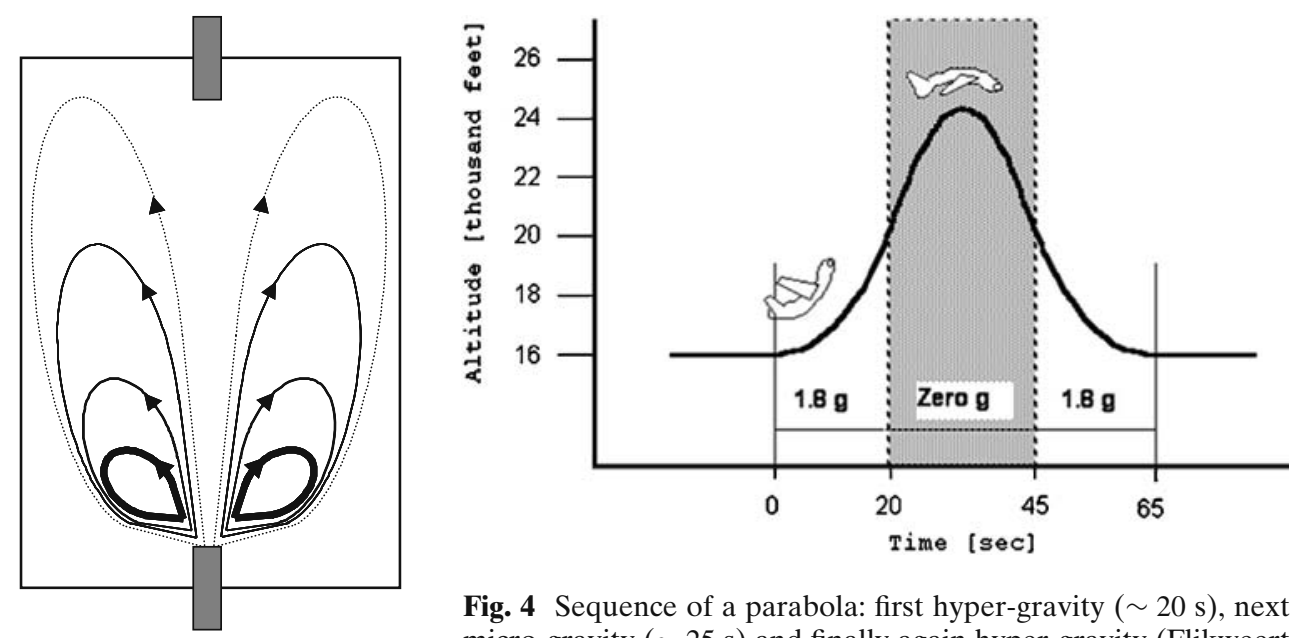

Fig. 4 Sequence of a parabola: first hyper-gravity $(\sim 20 \mathrm{~s})$, next micro-gravity $(\sim 25 \mathrm{~s})$ and finally again hyper-gravity (Flikweert et al. 2006; European Space Agency 2009)

convection is absent or when the convection is much stronger than diffusion, good mixing is observed: no axial colour segregation is seen in the lamp. Fischer (1976) developed a theory for an infinitely long lamp which gives the segregation as a function of the convection speed, which is depicted in Fig. 3. Because the convection is induced by gravity, measuring under different gravity conditions aids the understanding of the diff usive and convective processes.

\section{Parabolic Flights}

The measurements during the parabolic flights have been published before (Flikweert et al. 2006; Stoffels et al. 2005) and will be summarized here.

During the parabolic flights, the dynamic lamp behaviour has been investigated to obtain a relationship between the gravity and atomic Dy density in the lamp. The lamp was measured during the parabolic-

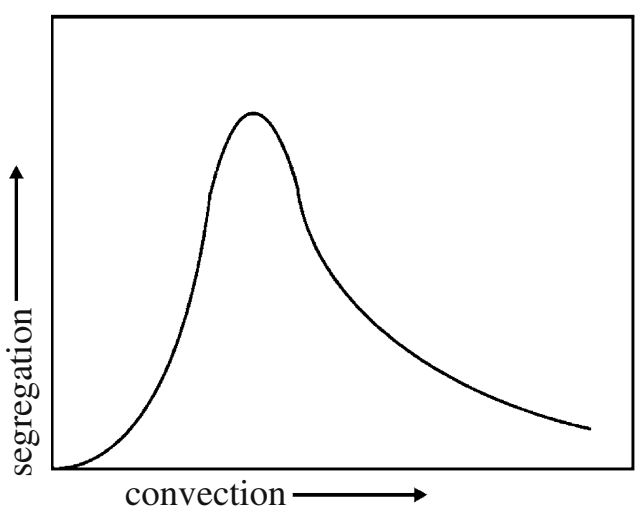

Fig. 3 The Fischer curve (Fischer 1976; Flikweert et al. 2006) which gives the axial segregation as a function of the convection speed. Convection is induced by gravity. At $1 g$ our lamps are around the maximum of the curve flight campaign of the European Space Agency (ESA) in June 2004 (European Space Agency 2009). During each flight, 31 parabolas with micro-gravity and hypergravity were performed. Figure 4 shows one parabola schematically.

In the setup we used laser absorption spectroscopy. The beam of a diode laser is expanded to a sheet. When it illuminates the lamp, part of the light is absorbed by the dysprosium atoms. After the lamp and optics the laser light hits a diode array, which measures the remaining intensity of the laser light. In this way, a line-of-sight density (column density) profile of atomic ground state dysprosium is obtained at one axial position of the lamp, which gives a measure for the amount of axial segregation. In addition, the lamp voltage has been measured. This voltage gives a measure for the total amount of dysprosium in the arc discharge.

The Dy density in the lamp and the voltage over the lamp (which is proportional to the total amount of Dy in the arc) are measured during a parabola. Figure 5 shows an example of the density and voltage of the lamp with $4 \mathrm{mg} \mathrm{DyI}$ and $10 \mathrm{mg} \mathrm{Hg}$, at $150 \mathrm{~W}$ input power. The values given in this graph are relative values; the atomic ground state Dy density is in the order of $10^{21} \mathrm{~m}^{-3}$ and the voltage is $\sim 80 \mathrm{~V}$. The Dy density varies with gravity and it is observed that the voltage follows the same trend as the Dy density. The increase in Dy density at the first hyper-gravity phase is explained by a better mixing and thus less axial segregation above $1 g$. This is because we are at the right hand of the Fischer curve (Fig. 3) (Flikweert et al. 2005, 2006; Nimalasuriya et al. 2006). However, it is seen that at the end of the micro-gravity phase, the Dy density is still not constant. This is because the diffusion time constant is greater than the $25 \mathrm{~s}$ of micro-gravity. 


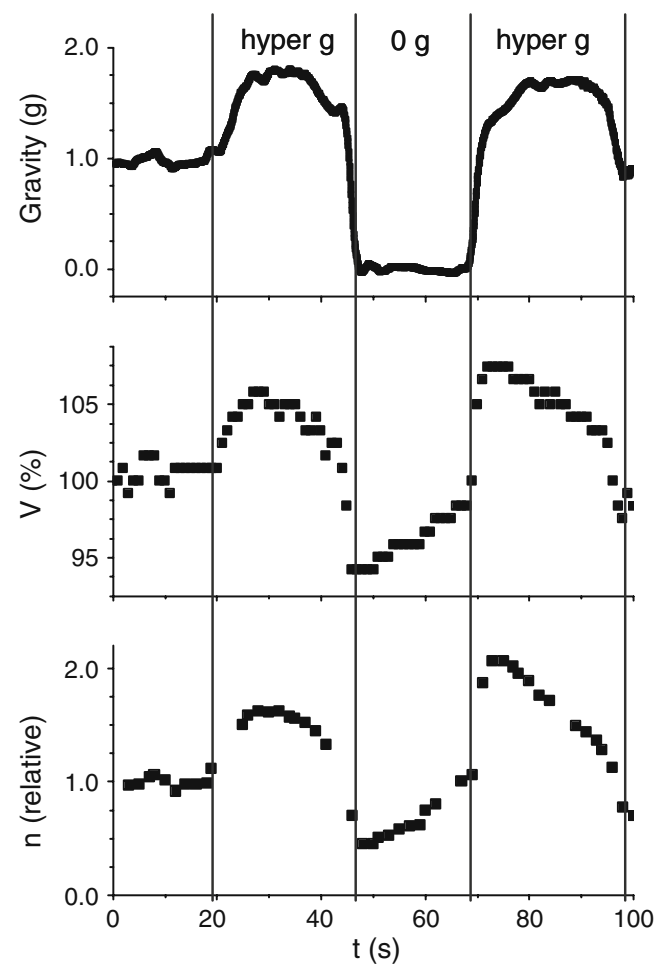

Fig. 5 Gravity, lamp voltage and radially averaged ground state atomic Dy density for a lamp with $10 \mathrm{mg} \mathrm{Hg}$ and $4 \mathrm{mg} \mathrm{DyI} \mathrm{I}_{3}$ during one parabola, as functions of time (Flikweert et al. 2006)

This problem does not occur in the ISS, where we have micro-gravity for a period much longer than during the parabolic flights.

Movie 1 shows a webcam movie of the lamp $(10 \mathrm{mg}$ $\left.\mathrm{Hg}, 4 \mathrm{mg} \mathrm{DyI})_{3}\right)$ during the parabola. The power $(P=$ $130 \mathrm{~W}$ ) is slightly lower than at the Dy density measurements. Note that the lamp is rotated by $90^{\circ}$; the right side in the movie corresponds to the bottom of the lamp whereas the left side corresponds to the top. The effects as described before are also observed in the movie: better mixing at $2 g$ and no axial segregation at $0 g$. It takes some time for the lamp to stabilize at $0 g$ due to the diffusion time constant as already explained.

For a thorough investigation of the lamp the periods are also too short. Therefore we built a centrifuge, where hyper-gravity can be prolonged. We can also go up until $10 \mathrm{~g}$, which allows us for measuring the whole range of the Fischer curve. We can also investigate other lamps that are much more at the left-hand side in the Fischer curve at $1 g$ (Fig. 3) than the lamp presented in this section.

\section{The Centrifuge}

A centrifuge was built to investigate the lamp under hyper-gravity conditions (Flikweert et al. 2007). The ar- tificial gravity that is achieved by the centrifuge ranges from $1 g$ to $10 g$.

Movie 2 shows a compilation of the centrifuge speeding up to $10 \mathrm{~g}$. The total time to reach the maximum speed takes about $3 \mathrm{~min}$; the authors have edited the movie so some shots are taken during speeding up. It is clearly seen that the gondola swings out while increasing the artificial gravity. In this way the artificial gravity vector always stays parallel to the lamp axis. Finally the centrifuge slows down again to $1 g$.

\section{System Overview}

A schematic representation of the centrifuge is given in Fig. 6. The centrifuge consists of a pivot, an arm and a gondola attached to the arm. The gondola contains all electrical equipment and the measurement setup (including the lamp) itself. The total diameter at maximum swing-out of the gondola is about $3 \mathrm{~m}$. The maximum rotation speed is $\sim 1.5 \mathrm{~Hz}$ which corresponds to $50 \mathrm{~km} \mathrm{~h}^{-1}$. The maximum payload is $100 \mathrm{~kg}$. The gondola swings out around it hinge point when spinning the centrifuge, so the artificial gravity vector is always parallel to the lamp axis, as is shown schematically in Fig. 7. In this figure, $\mathbf{g}_{z}$ is the acceleration due to earth's gravity, $\mathbf{a}_{r}$ is the centrifugal acceleration and $\mathbf{a}_{\text {tot }}$ is the total acceleration experienced by the lamp. The arm

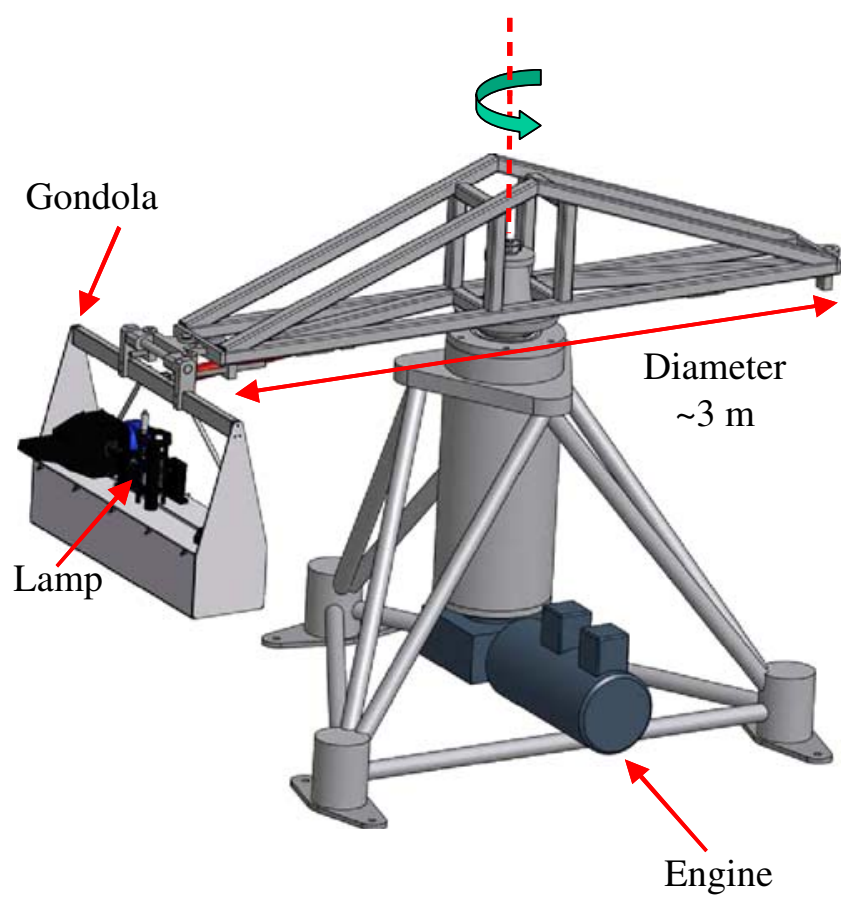

Fig. 6 Schematic representation of the centrifuge. It consists of a pivot, an arm and the gondola that contains the lamp and diagnostics (Flikweert et al. 2007) 


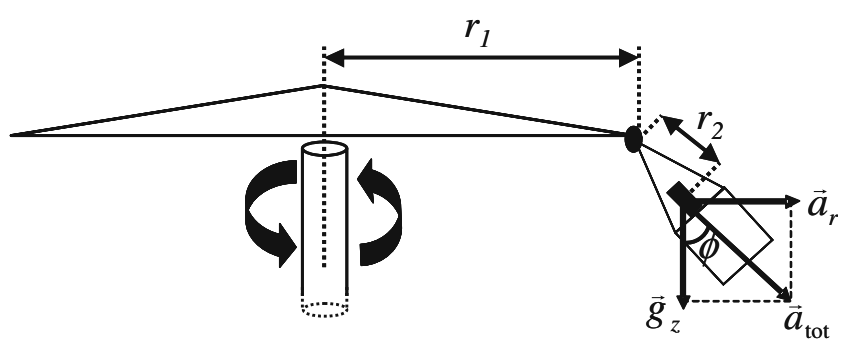

Fig. 7 Schematic representation of the centrifuge arm with the gondola. When rotating, the gondola swings out with an angle $\phi$. The acceleration vectors at the position of the lamp are indicated (Nimalasuriya et al. 2007; Flikweert et al. 2007)

length is $r_{1}=1.10 \mathrm{~m}$ and the distance from the hinge point to the lamp centre is $r_{2}=0.35 \mathrm{~m}$. Because the top of the lamp is closer to the centrifuge centre than the bottom of the lamp, a slight variation in gravity over the lamp occurs. This variation is at most $0.7 \%$ at $10 g$.

Because of the convection flows inside the lamp, the moving Dy particles experience a Coriolis acceleration

$\mathbf{a}_{\mathrm{cor}}=-2 \omega \times \mathbf{v}$,

where $\boldsymbol{\omega}$ is the angular speed vector of the centrifuge and $\mathbf{v}$ is the flow speed at the axis. The angular speed $|\boldsymbol{\omega}|$ is given by Flikweert et al. (2007)

$\omega=\left(\sqrt{a_{\mathrm{tot}}^{2}-g_{z}^{2}} / r\right)^{\frac{1}{2}}$

and $|\mathbf{v}|$ at the axis is $v=\left(a_{\text {tot }} / g_{z}\right) \times 0.2 \mathrm{~m} \mathrm{~s}^{-1}$ (Beks 2008). This gives $a_{\text {cor }}=3 g_{z}$ under the artificial gravity of $10 \mathrm{~g}(r=1.45 \mathrm{~m})$, where it acts perpendicular to the lamp axis.

The measurement system in the centrifuge is shown in Fig. 8. We use a control PC outside the centrifuge lab. This PC drives the frequency controller of the centrifuge engine. The gondola contains another (mini-) computer on which the experimental software is running. The experimental software is written in NI CVI LabWindows. In addition to this computer, the gondola contains all the equipment: the lamp power supply, a CCD camera, an ordinary webcam, the laser controller and a data acquisition system. The experiment itself is on the rail on top of the gondola. The computer inside the gondola is connected to the control PC by the wireless network. The only fixed connection (slip ring) to the outer world is the $230 \mathrm{~V}$ power wire. We use Windows Remote Desktop to operate the gondola PC. During the measurements, the gondola PC collects the data on its flash disks. After the measurements are finished, the data is transferred to the control PC and the data is processed off-line.

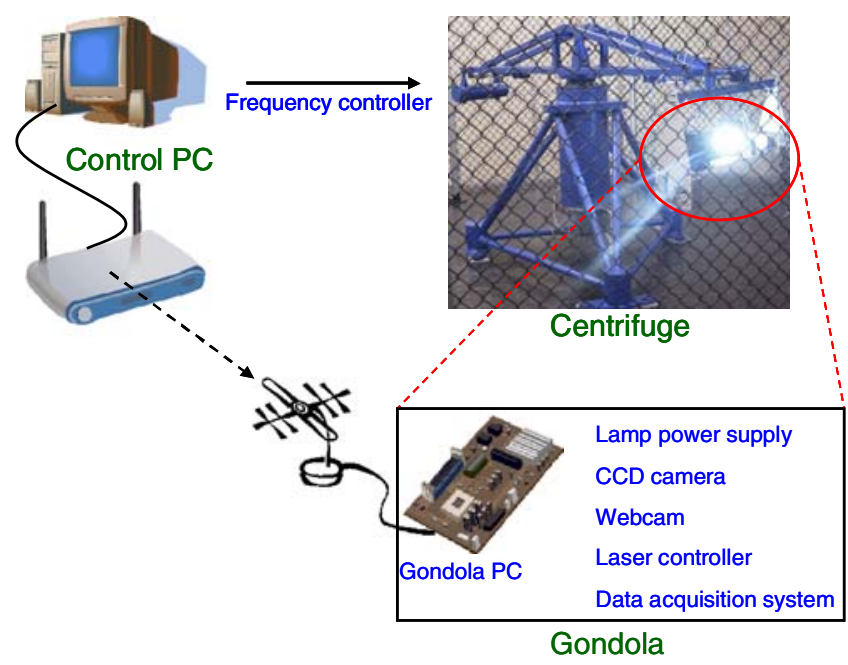

Fig. 8 The measurement system in the centrifuge. The equipment in the centrifuge gondola is controlled by a mini-computer, which is connected via wireless network to the control PC

The measurement techniques include:

- webcam imaging: an ordinary webcam monitors the lamp, which gives an indication of the axial segregation by eye;

- emission spectroscopy: gives line intensities of $\mathrm{Hg}$ and Dy, from which temperature profiles and densities can be derived;

- ILAS: gives a 2D density profile of atomic ground state Dy.

\section{Webcam Images}

The lamp is monitored on-line by an ordinary webcam on top of the gondola in the centrifuge. From the webcam images, colour segregation can be observed clearly. Figure 9 shows various webcam images of the lamp with $10 \mathrm{mg} \mathrm{Hg}$ and $4 \mathrm{mg}$ of $\mathrm{DyI}_{3}$ (Flikweert et al. 2008). The input power is $150 \mathrm{~W}$. A picture taken at the ISS $(0 g)$ is displayed for comparison. The other images are taken at the centrifuge setup. At $0 g$ no axial segregation is present; we are at the origin of the Fischer curve (Fig. 3). At $1 g$ we are in the area of the maximum of the curve. Increasing the gravity means increasing the convection and thus moving to the right on the Fischer curve. This is observed in the pictures: when gravity is increased, better mixing occurs and axial segregation is diminished.

\section{Emission Spectroscopy}

The lamp was investigated by means of emission spectroscopy, which gives the line intensities of $\mathrm{Hg}$ and $\mathrm{Dy}$. 
Fig. 9 Pictures of the lamp at $0 g-10 g$. The first image is taken in the ISS

(Nimalasuriya et al. 2006), the others are taken in the centrifuge setup (Flikweert et al. 2007, 2008). Note that the exposure time of the ISS picture is different from those at the centrifuge

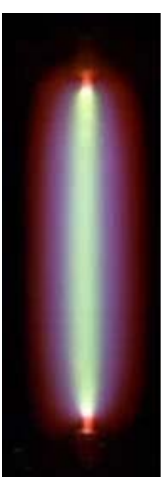

$0 \mathrm{~g}$ (ISS)

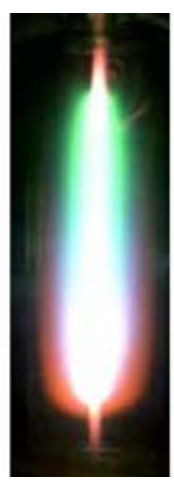

$1 g$

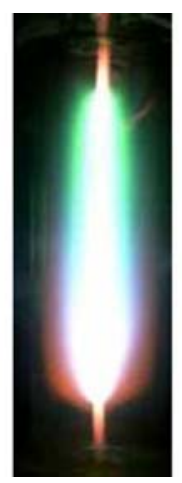

$2 g$

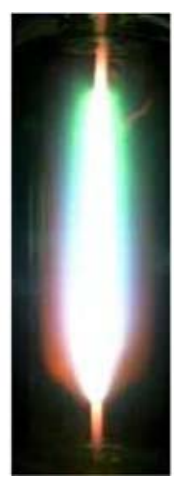

$4 g$

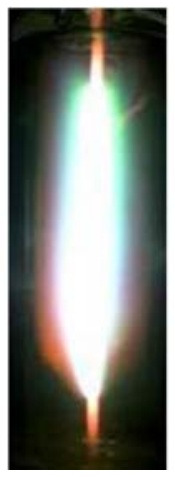

$6 g$

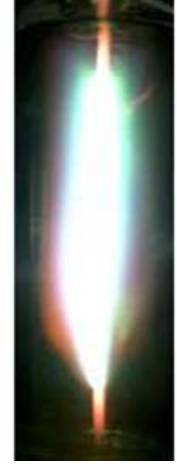

$8 g$

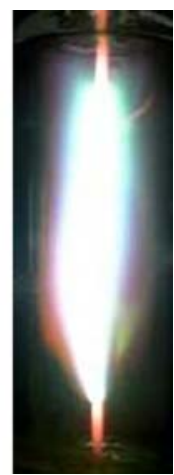

$10 \mathrm{~g}$
From a calibrated $\mathrm{Hg}$ line the temperature profile of the lamp was obtained. After combining these temperature profiles with the calibrated Dy lines, assuming Local thermodynamic equilibrium (LTE), we get absolute Dy densities (Nimalasuriya 2007; Nimalasuriya et al. 2006). The spectrometer used for the optical emission measurements was originally designed for the ISS: it has to be compact and light-weighted. These requirements were met using a Echelle spectrometer. The light from the slit of the spectrometer is dispersed by the grating and reflected back on a CCD camera. The image contains the wavelength in horizontal direction and the lateral position in the lamp in vertical direction. In this way we obtain a spectrum for each lateral position in the lamp, at a chosen axial position in the lamp.

The axial segregation that was observed by the webcam (Fig. 9) is also observed by emission spectroscopy. In Fig. 10 the intensity profiles of a Dy line are shown as a function of the lateral position in the lamp. These profiles are obtained at 3 axial positions in the lamp (10 mg Hg, $4 \mathrm{mg} \mathrm{DyI}_{3}, P=150 \mathrm{~W}$ ), for both normal gravity $(1 g)$ and $10 g$. Note that the intensity profiles and webcam images were taken from different angles $\left(90^{\circ}\right.$ with respect to each other) (Flikweert et al. 2007). From the profiles at the bottom, we see that the amount of Dy decreases from $1 g$ to $10 g$. At the top we see an increase of the amount of Dy when increasing $g$; Dy is mixed better over the lamp (less axial segregation). The decreased Dy intensity near the bottom at $10 \mathrm{~g}$ can be explained by a lower cold spot temperature; this spot is located at the bottom of the lamp. This decrease is explained by two effects. Firstly, because of arc contraction the wall becomes cooler and subsequently the cold spot temperature decreases. Secondly, because of better mixing the power is distributed more evenly over the lamp. This causes a lower power density at the bottom compared to the $1 g$ situation and thus a lower cold spot temperature (Nimalasuriya 2007; Nimalasuriya et al. 2007).

\section{Imaging Laser Absorption Spectroscopy}

In addition to emission spectroscopy, the lamp was measured by using the Imaging Laser Absorption

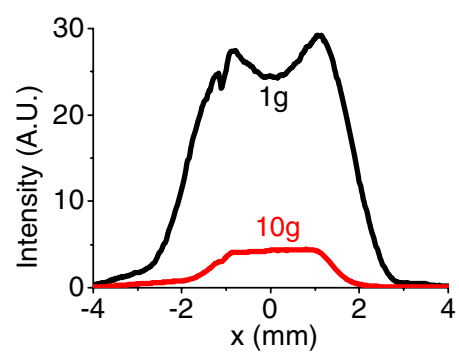

(a)

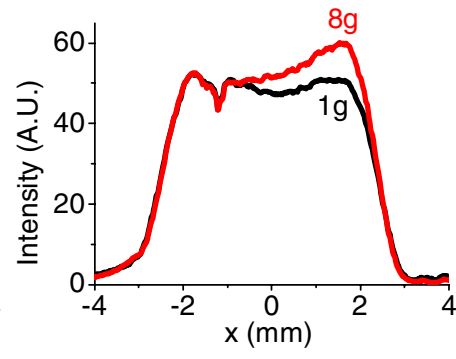

(b)

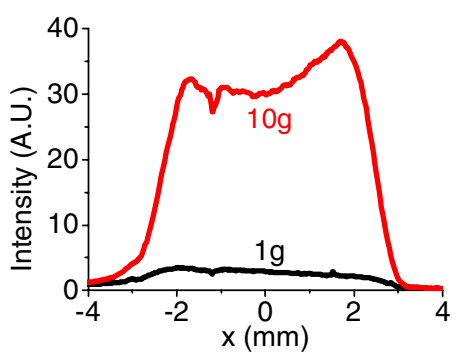

(c)

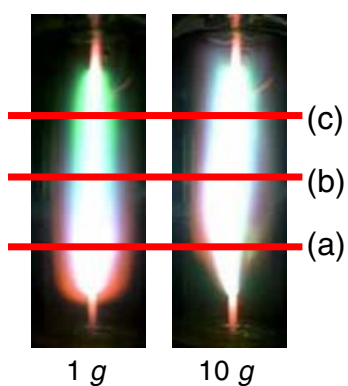

(d)
Fig. 10 Atomic Dy line $(642.19 \mathrm{~nm})$ intensities at different axial positions in the lamp measured by emission spectroscopy, for normal and hyper-gravity (Nimalasuriya et al. 2007): a bottom; b centre; $\mathbf{c}$ top. In $\mathbf{d}$ the webcam images are given for comparison; the positions where the profiles were taken are indicated 
Spectroscopy (ILAS) technique (Flikweert et al. 2007). ILAS yields the 2D density distribution of ground state atomic Dy. We use a diode laser (line width $\leq$ $3 \mathrm{fm})$ and scan around an absorption line of atomic Dy $(642.19 \mathrm{~nm})$, which has a line width of $\sim 40 \mathrm{pm}$ (Flikweert et al. 2005, 2006). The laser beam is expanded so it illuminates the whole lamp burner. While passing the lamp, the Dy atoms in the lamp absorb part of the beam. After the lamp and optics, the laser light is imaged on a CCD camera. In this way, for each position in the lamp an absorption profile is constructed. From these profiles the atomic ground state Dy density is calculated so a $2 \mathrm{D}$ density plot is obtained.

The 2D density of ground state atomic Dy is shown for $1 g$ and $10 g$ in Fig. 11. The lamp filling is $5 \mathrm{mg}$ $\mathrm{Hg}$ and $4 \mathrm{mg} \mathrm{DyI}{ }_{3}$; the input power is $150 \mathrm{~W}$. These density profiles show that a better mixing occurs at $10 \mathrm{~g}$ : the amount of Dy at the top of the lamp is increased. This is consistent with the webcam images in Fig. 9 and with the emission measurements in Fig. 10, which show also a better mixing and thus less colour segregation at higher gravity. The ilas pictures also show a decrease in Dy density near the bottom of the lamp at $10 \mathrm{~g}$, which is caused by the decrease in cold spot temperature as explained in section Emission Spectroscopy. For a comparison between the experimental ILAS results and numerical results obtained by modelling the reader is referred to literature (Flikweert et al. 2008; Beks et al. 2006, 2008).

When we compare the methods of emission spectroscopy and ILAs, both derive lateral atomic Dy density profiles. Emission spectroscopy derives the atomic $\mathrm{Hg}$

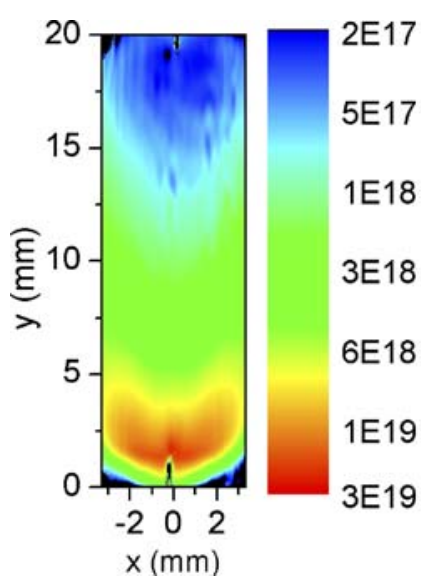

(a)

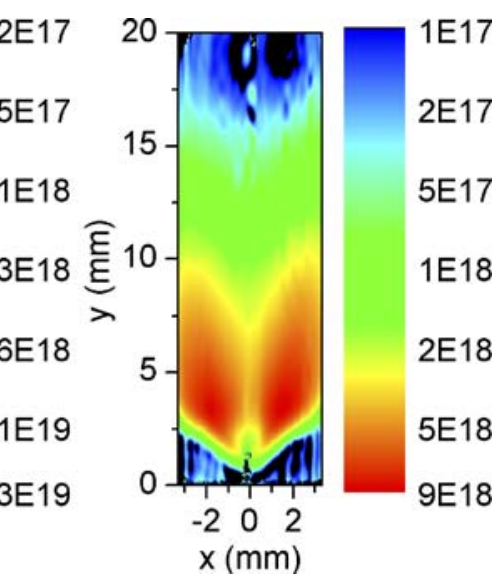

(b)
Fig. 11 Atomic ground state Dy density (column density in $\mathrm{m}^{-2}$ ) measured by ILAs (Flikweert et al. 2007), $P=150 \mathrm{~W}, 5 \mathrm{mg} \mathrm{Hg}$ and $4 \mathrm{mg} \mathrm{DyI}_{3}$, a $1 \mathrm{~g}$; b $10 \mathrm{~g}$. Note that the flame-like pattern at the top of the lamp is an artefact caused by the data analysis and atomic and ionic Dy density, and temperature. The setup needs to be calibrated to obtain absolute line intensities and densities. Local thermodynamic equilibrium (LTE) (van der Mullen 1990) is assumed. ILAS derives the ground state atomic Dy density directly and a 2D profile is obtained directly. With ILAs, also nonradiating Dy atoms can be measured in contrast to the emission spectroscopy setup. This means that by using ILAS we can also measure the colder regions in the lamps where emission spectroscopy cannot detect the Dy atoms. To obtain the total atomic Dy density (not only ground state) from ILAS, the temperature has to be estimated (Beks et al. 2008). Note that the density plots shown are line-of-sight (column) densities. In future these will be converted into radial profiles by means of Abel inversion (Bakker 2000; Buie et al. 1996; Pretzler et al. 1992; Sudharsanan 1986; Kak and Slaney 1988).

\section{Conclusions}

The metal-halide lamp shows colour segregation, which is caused by the combination of diffusion and convection. We used artificial gravity to change the amount of convection and study the flow phenomena in the lamp. Beside the lab measurements $(1 g)$ this was done at the ISS $(0 g)$, parabolic flights $(0 g$ and $2 g)$ and in a centrifuge $(1 g-10 g)$. The three measurement methods (a webcam, emission spectroscopy and ILAs) all show that in the lamp we used, enhancing gravity and thus increasing the amount of convection diminishes axial segregation. The webcam images show that axial colour segregation is diminished. By making use of varying gravity conditions the whole Fischer curve can be measured.

In the near future we want to have a closer look at the ILAS measurements and to obtain several physical parameters from these measurements. Furthermore the emission spectroscopy measurements will be extended to axial intensity measurements, from which we can deduce the axial segregation parameter directly from the line intensities. This will enable us to measure also other elements than Dy and for measuring commercially available lamps.

Acknowledgements The authors are grateful to A.F. Meunier and G.M. Thubé, the General Technical Department of the Eindhoven University of Technology, Philips ADL, SenterNovem (project EDI 03146), SRON and the Dutch Ministries of Research and Education as well as Economic Affairs for funding the research.

Open Access This article is distributed under the terms of the Creative Commons Attribution Noncommercial License which 
permits any noncommercial use, distribution, and reproduction in any medium, provided the original author(s) and source are credited.

\section{References}

Bakker, L.P.: Plasma Control of the Emission Spectrum of Mercury-Noble-gas Discharges. Ph.D. thesis, Eindhoven University of Technology (2000)

Beks, M.L.: Modelling Additive Transport in Metal Halide Lamps. $\mathrm{Ph} . \mathrm{D}$. thesis, Eindhoven University of Technology (2008)

Beks, M.L., Hartgers, A., van der Mullen, J.J.A.M.: Demixing in a metal halide lamp, results from modelling. J. Phys. D: Appl. Phys. 39, 4407 (2006)

Beks, M.L., Flikweert, A.J., Nimalasuriya, T., Stoffels, W.W., van der Mullen, J.J.A.M.: Competition between convection and diffusion in a metal halide lamp, investigated by numerical simulations and imaging laser absorption spectroscopy. J. Phys. D 41, 144025 (2008)

Bellows, A.H., Feuersanger, A.E., Rogoff, G.L., Rothwell, H.L.: HID convection studies: a space shuttle experiment. In: Illuminating Engineering Soc. Meeting, St Louis, 5-9 August 1984

Bellows, A.H., Feuersanger, A.E., Rogoff, G.L., Rothwell, H.L.: Convection and additive segregation in high pressure lamp arcs: Early results from a space shuttle experiment. Gaseous Electronics Conf. Bull. Am. Phys. Soc. 30, 141 (1985)

Bonvallet, G.A., Lawler, J.E.: Optical absorption spectroscopy on a metal-halide high intensity discharge arc lamp using synchrotron radiation. J. Phys. D 36, 1510-1518 (2003)

Buie, M.J., Pender, J.T.P., Holloway, J.P., Vincent, T., Ventzek, P.L.G., Brake, M.L.: Abel's inversion applied to experimental spectroscopic data with off axis peaks. J. Quant. Spectrosc. Radiat. Transfer 55, 231-243 (1996)

Dakin, J.T., Shyy, W.: The prediction of convection and additive demixing in vertical metal halide discharge lamps. J. Electrochem. Soc. 136, 1210-1214 (1989)

Dakin, J.T., Rautenberg Jr., T.H., Goldfield, E.M.: Anatomy of a vertical metal halide discharge. J. Appl. Phys. 66, 4074-4088 (1989)

European Space Agency: European Space Agency homepage. http://www.esa.int (2009)

Fischer, E.: Axial segregation of additives in mercury-metalhalide arcs. J. Appl. Phys. 47, 2954-2960 (1976)

Flikweert, A.J., Nimalasuriya, T., Groothuis, C.H.J.M., Kroesen, G.M.W., Stoffels, W.W.: Axial segregation in high intensity discharge lamps measured by laser absorption spectroscopy. J. Appl. Phys. 98, 073301 (2005)

Flikweert, A.J., van Kemenade, M., Nimalasuriya, T., Haverlag, M., Kroesen, G.M.W., Stoffels, W.W.: Axial segregation in metal-halide lamps under varying gravity conditions during parabolic flights. J. Phys. D 39, 1599-1605 (2006)

Flikweert, A.J., Nimalasuriya, T., Kroesen, G.M.W., Stoffels, W.W.: Imaging Laser Absorption Spectroscopy of the metalhalide lamp in a centrifuge (1-10g). Plasma Sources Sci. Technol. 16, 606-613 (2007)

Flikweert, A.J., Beks, M.L., Nimalasuriya, T., Kroesen, G.M.W., van der Mullen, J.J.A.M., Stoffels, W.W.: 2-D images of the metal-halide lamp obtained by experiment and model. IEEE Trans. Plasma Sci. 36, 1174-1175 (2008)

International Energy Agency: Light's Labour's Cost: Organisation for economic co-operation and development. ISBN 9264-10951-X. http://www.iea.org (2006)
Kak, A.C., Slaney, M.: Principles of Computerized Tomographic Imaging. IEEE, Piscataway (1988)

Lister, G.G., Lawler, J.E., Lapatovich, W.P., Godyak, V.A.: The physics of discharge lamps. Rev. Mod. Phys. 76, 541-598 (2004)

Nimalasuriya, T.: Transport Phenomena in Metal-Halide Lamps, a poly-diagnostic study. Ph.D. thesis, Eindhoven University of Technology (2007)

Nimalasuriya, T., Curry, J.J., Sansonetti, C.J., Ridderhof, E.J., Flikweert, A.J., Stoffels, W.W., Haverlag, M., van der Mullen, J.J.A.M.: X-ray induced fluorescence measurement of the density distribution of Dy and $\mathrm{Hg}$ in a metal-halide lamp. In: XXVIIth International Conference on Phenomena in Ionized Gases (2005)

Nimalasuriya, T., Flikweert, A.J., Haverlag, M., Kemps, P.C.M., Kroesen, G.M.W., Stoffels, W.W., van der Mullen, J.J.A.M.: Metal halide lamps in the international space station ISS. J. Phys. D 39, 2993-3001 (2006)

Nimalasuriya, T., Pupat, N.B.M., Flikweert, A.J., Stoffels, W.W., Haverlag, M., van der Mullen, J.J.A.M.: Optical emission spectroscopy of metal-halide lamps: radially resolved atomic state distribution functions of dy and hg. J. Appl. Phys. 99, 053302 (2006)

Nimalasuriya, T., Thubé, G.M., Flikweert, A.J., Haverlag, M., Kroesen, G.M.W., Stoffels, W.W., van der Mullen, J.J.A.M.: Axial segregation in metal-halide lamps under varying gravity conditions ranging from 1 to $10 \mathrm{~g}$. J. Phys. D 40, 2839-2846 (2007)

Pretzler, G., Jäger, H., Neger, T., Philipp, H., Woisetschläger, J.: Comparison of different methods of abel inversion using computer simulated and experimental side-on data. Z. Naturforsch. 47a, 955-970 (1992)

Rogoff, G.L., Feuersanger, A.E., Bellows, A.H., Rothwell, H.L.: Convection and additive segregation in metal-halide lamp arcs: results from a space shuttle experiment. In: Symp. on Science and Technology of High Temperature Light Sources, Electrochemical Society Meeting, vol. 85-1, pp. 551-552, Toronto, May (1985)

Rogoff, G.L., Feuersanger, A.E., Drummey, J.P., Rothwell Jr., H.L.: Determination of two-dimensional temperature and additive density distributions in a high-intensity-discharge lamp arc. J. Appl. Phys. 62, 4084-4089 (1987)

Stoffels, W.W., Kemps, P.C.M., Beckers, J., Kroesen, G.M.W., Haverlag, M.: Light emission of metal halide lamps under mirco- and hypergravity conditions. Appl. Phys. Lett. 87, 241501 (2005)

Stoffels, W.W., Baede, A.H.F.M., van der Mullen, J.J.A.M., Haverlag, M., Zissis, G.: Definition of a high intensity metal halide discharge reference lamp. Meas. Sci. Technol. 17, N67 (2006)

Stoffels, W.W., Nimalasuriya, T., Flikweert, A.J., Mulders, H.C.J.: Discharges for lighting. Plasma Phys. Control. Fusion 49, B505-B512 (2007)

Stormberg, H.-P.: Axial and radial segreagation in metal halide arcs. J. Appl. Phys. 52, 3233-3237 (1981)

Sudharsanan, S.I.: The Abel Inversion of Noisy Data Using Discrete Integral Transforms. Ph.D. thesis, Knoxville: University of Tennessee (1986)

van der Mullen, J.A.M.: Excitation equilibria in plasma's; a classification. Phys. Rep. 191, 109-220 (1990)

Zhu, X.: Active spectroscopy on HID lamps-exploration of various methods. Ph.D. thesis, Eindhoven University of Technology (2005)

Zollweg, R.J.: Reducing additive segregation in metal halide high-intensity discharge lamps. J. IES 5, 12-19 (1975) 